3. die Darstellung ron Calciumborid aus Boroxgd und Calciumcarbid wahrscheinlich gemacht;

4. bei Borstickstoff der Beginn der Abgabe von Slickstoff im Folulensuliffehen bei ea. $2450^{\circ}$ festgestellt;

5. aus Boroxyd, Koble und Stickstoff bei einem Temperaturoptimum ron $1,50-1700^{\circ}$ unter gewöhnlichem Druck in maximo $26-2 \mathrm{~S} \% \mathrm{BN}$, unter hiiherem Druck jedoch mehr als $85 \%$ BN erhalten;

6. aus Borocalcit, lioble und Stickstoft bei $1800^{\prime \prime} \rightarrow 1400^{\prime \prime}$ naliczu die theoretische Ausheute $(46 \%)$ an gebundenem Stickstoff erhalten, wobei eine Druckerhöhung ohne jeden Eiofluß Uleibt;

†. ein clektrischer Druckofon konstruiort für Drucke bis zn $500 \mathrm{~kg}$ pro $1 \mathrm{~cm}$ und Temperaturen bis zu cal $2500^{\circ}$.

Die Untersucbung soll in änlicher Weise auf die Synthese von Cyaniden alusgedebut werden. Zur Beschaffung der - auch anderen 7wecken dienenden - Iochdruckapparatur standen uns zum leil Nittel aus der städtischen Jagor-Stiftung zı Berlin zur Verfügung, wolür auch an dieser Stelle verbindlichst gedankt sei.

263. Wilhelm Traube und Richard Ascher:

Über das Isohydantoln 2-Imino-4-keto-tetrahydro-oxazol und seine Homologen.

[Aus dem Chemischen Institut der Universität Berlin.]

(Eingegangen am 12. Juni 1913.)

Guanidin wirkt, wie der eine von uns kürzlich gezeigt hat'), auf die Ester der Fettsäuren und der $\alpha$-halogen-substituierten Fettsäuren unter Abspaltung von Alkohol und Bildung ron Acidylguanidinen eis.

Etwas komplizierter verläuft die Reaktion zwischen Guanidin

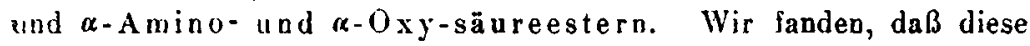
Jister mit der Base nicht nur Alk ohol abspalten, sondern daß bei der Reaktion gleichzeitig Ammoniak in Freibeit gesetzt wird.

Aus Guanidin und Glykokollester entsteht so Glykocyamidin, iudem die Reaktion, wie man anuelımen muß, in zwei Phasen verläuft. Es bildet sich zuerst [Amino-ncetyl]-guanidin, das daun unter Verlust von Ammoniak und Schließung des Glyoxaliuringes in das Glykocyamidin übergeht:

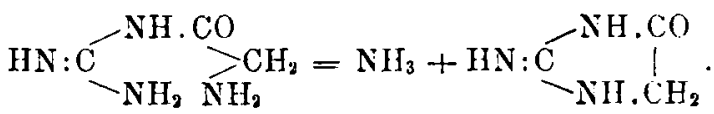

ग) B. 43, $3586[1910]$. 
Bei der Reaktion zwischen Guanidin und $\propto$-Oxysäureestern, z. B. Gijkolsäureester, entsteht dementsprechend zweifellos zuerst ebenfalls als $Z$ wischenprodukt ein einfaches Acidylderivat der Base, das $\left[\mathrm{O}\right.$ y-acetyl]-guanidin, $\mathrm{NH}_{2} . \mathrm{C}(\mathrm{NH}) \cdot \mathrm{NH} . \mathrm{CO} \cdot \mathrm{CH}_{2} . \mathrm{OH}$.

Wenn dann aus diesem letzteren Ammoniak eliminiert wird, so sind zwei Möglichkeiten gegeben; es kann entweder ein CyanamidDerivat (Formel I) oder aber ein ringförmig konstituierter Körper, ein Derivat eines hydrierten Oxazols entstehen (Formel II):

$$
\begin{gathered}
\mathrm{HO} . \mathrm{CH}_{2} . \mathrm{CO} . \mathrm{NH} . \mathrm{CN} \quad \mathrm{HN}: \mathrm{C}-\mathrm{NH} . \underset{\mathrm{CO}}{\mathrm{C}}-\underset{\mathrm{CH}}{\mathrm{C}}, \\
\text { I. }
\end{gathered}
$$

in welch letzterem Falle die Ammoniakabspaltung zwischen der Aminound der Hydroxylgruppe des Oxyacetyl-guanidins erfolgt.

Da: wie festgestellt wurde, der in der Hydroxylgruppe äthylierte Glykolsäureester, der Äthoxy-essigsäureester mit Guanidin glatt unter Bildung nur von $[\ddot{t}$ thoxy-acet $y \mathrm{~J}]$-guanidin, $\mathrm{C}_{2} \mathrm{H}_{5} \mathrm{O} . \mathrm{CH}_{2} . \mathrm{CO}$. NH.C(:NH). $\mathrm{NH}_{2}$, reagiert, und da andererseits auch dieses letztere vicht zur Ammoniakabspaltung neigt, so war es von vornherein wahrscheit:lich, da $B$ bei der Ammoniak-Abspaltung aus dem Oxyacetyl-guanidin die ireie Hydroxylgruppe dieses letzteren beteiligt ist, daß die Reaktion also unter Ringscbluß erfulgt in analoger Weise wie bei dem oben erwähnten Übergange des Aminoacetyl-guanidins in Glyk cyamidin.

Die chemischen Eigenschaften des aus der Realtion zwischen Guanidin und Glykolsäureester hervorgehenden Körpers lassen vollends keinen Zweifel, daß ein der Formel II entsprechender Körper, d. b. ein 2-Imino-4-keto-tetrahydro-oxazol vorliegt.

Dasselbe ist einerseits ein Analogon des Maly schen Pseudothiohydantoins, andererseits ein Isomeres des Baeyerschen $\mathrm{Hydau}$ toins, wesbalb man es auch als Iso-oder Pseudo-bydantoin bezeicbnen kann.

Das Isobydantoin ist eine schwache Base, die in der $\mathrm{K}$ älte mit Mineralsäuren gut krystallisierende Salze bildet. Beim Erwärmen nit Säuren erfolgt dagegen eine Reaktion, die völlig derjenigen entspricht, die bei der Einwirkung von Säuren auf Pseudothiohydantoin vor sich geht. Es spaltet sich in beiden Fällen hydrolytisch Ammoniak ab, indem bekanntlich aus dem Pseudothio-bydantoin die sogenannte Senfölessigsäure, das Diketo-tetrahydrothiazol sich bildet, während aus dem hier in Rede stehenden $\mathbf{I}_{\text {so }}$ bydantoin ein Körper der Formel $\mathrm{O}: \mathrm{C}<\underset{\mathrm{O}-\mathrm{CH}_{3}}{\mathrm{NH}}$ ein Diketo-tetrahydro-oxazol entsteht. Dieses letztere, ein bei $89-90^{\circ}$ schmel- 
zender und unter vermindertem Druck unzersetzt destillierender Körper, stellt mithin das sauerstoffhaltige Analogon der Sedföl-essigsäure dar.

Die so leicht unter dem Einflusse von Säuren erfolgende bydrolytische Ammoniak-Abspaltung würde, wie hervorgehoben sei, nicht zu deuten sein, wenn man dem aus Guanidin und Glykolsäureester entstehenden Körper die obige Formel I, d. b. die Formel eines Oxyacetyl-cyanamids ${ }^{1}$ ) zuerteilen würde.

Die Ilomologen des Isobydantoins entstehen aus Guanidin und den Estern der Homologen der Glykolsäure. Wir haben unter Verwendung des Milchsäure- und Mandelsäureesters das Metb ylund Phenyl-isobydantoin dargestellt und beide in die entsprechenden Diketo-tetrahydro-oxazole übergeführt.

Ein [Oxy-metbyl]-isohydantoin haben wir durch Einwirkung run Guanidin anf Glycerinsäureester gewonnen.

Isohydantoin (2-Imino-4-keto-tetrabydro-oxazol), $\mathrm{C}_{3} \mathrm{H}_{4} \mathrm{~N}_{3} \mathrm{O}_{2}$.

Zur Gewinnuug des Isohydantoins werden $20 \mathrm{~g}$ Glykolsăureester mit etwa der berechneten Menge 50-prozentiger alkoholischer Guanidinlösung versetzt, wonach alsbald lebhafte Erwärmung und Entwieklung von Ammonials einsetzt. Nach kurzer Zoit beginnen Krystallnadeln sich abzuscheiden, lis schlieBlich das Gemisch zu einem festen Brei crstarrt. Die Krystalle werden abgesaugt, mit Alkohol gewaschen und dann aus etwa der 10-fachen Menge verdünnten Alkohols umkrystallisiert.

Die Ausbeuten an Rohprodukt schwanken zwischen 40 und $50 \%$ der theoretisch berechneten.

Der aus Alkohol in großen, starkbrechenden Prismen krystallisierende Körper beginnt bei $240^{\circ}$ zu sintern und schmilzt bei $246-247^{\circ}$ unter völliger Zersetzung.

$0.1393 \mathrm{~g}$ Sbst.: $0.1835 \mathrm{~g} \mathrm{CO}_{2}, 0.0497 \mathrm{~g} \mathrm{H}_{2} \mathrm{O} .-0.1543 \mathrm{~g}$ Sbst.: $37.5 \mathrm{~cm}$ $\mathrm{N}\left(20^{\circ}, 750 \mathrm{~mm}\right)$.

$$
\begin{aligned}
& \mathrm{C}_{3} \mathrm{H}_{4} \mathrm{~N}_{2} \mathrm{O}_{2} \text {. Ber. C } 36.00, \mathrm{H} 4.00, \mathrm{~N} 28.00 \text {. } \\
& \text { Gef. • 35.93, * 3.99, 》27.61. }
\end{aligned}
$$

Das Isohydantoin ist in Wasser, besonders in der Wärme, sehr leiclat löslich. Absoluter Alkohol löst es schwer und die übrigen gewöbnlichen organiscben Lösungsmittel so gut wie gar vicht.

1) Ein der obigen Formel I entsprechender Körper bezw. das nächste Homologe dieses Körpers, clas Cyanamid-Derivat der Milchsäure, HO. $\mathrm{CH}\left(\mathrm{CH}_{3}\right)$.CO.NH.CN, liegt nach den Untersuchungen von Mertens (J. pr. [?] 17, 34) in einer Verbindung vor, die bei der Finwirkung von Cyanamid auf Lactid bei Gegenwart von Kalium- oder Natriumäthylat entsteht. Ob diese Verbindung tatsächlich ein Derivat des Cyanamids, oder ob sie etwa identisch ist mit dem hier weiter unten beschriebenen Methyl-isohydantoin, welches wir aus Guanidin und Milchsaureester dargestellt halen, müßte noch untersucht werden. 
Auf Lackmuspapier reagiert die wäßrige Lösung neutral; doch ist die Substanz offeubar basisch und es gelang, auf folgendem Wege eiu gut lirystallisierendes salzsaures Salz zu erbalten.

$1 \mathrm{~g}$ Isohydantoin wurde in einem Überschusse alkoholischer Salzsïure retlisst und die Lösung im Exsiccitor über Natronkalk vordunstet. Hierbei suhied sich das Chlorhydrat in rhombischen Tafeln ab, die bei $152^{9} \%$ u sintern leggannen und bei $164^{\circ}$ unter Zersctzung schmolzen.

(0.1410 gr Sust.: $24.7 \mathrm{~cm} \mathrm{~N}\left(18^{n}, 760 \mathrm{~mm}\right) .-0.1991 \mathrm{~g}$ Sbst : $0.2140 \mathrm{~g} \mathrm{dgCl}$. $\mathrm{C}_{3} \mathrm{H}_{4} \mathrm{~N}_{2} \mathrm{O}_{9}, \mathrm{HCl}$. Ber. $\mathrm{N} 20.53, \mathrm{Cl} 25.97$.

Gef. " 20.2.2, > 26.43 .

Anlererseits bildet das Isohydantoin auch mit einiren Schwermetall en Sialze. Lügt man zu der aumoniabalischen Lüsung des Körpers eine ehensuche ron Silbernitrat, so fallt eine in riel heiBen Wasser lösliche Silberverbindung aus. Ammoniakalische Kupferlüsung gibt cine lichllban gefirbte liallung des linpfersalzes des Isohydantoins.

Durch Kochen mit Barytwasser wird das Isohydantoin in Ammoniak, Koblensäure und Glykolsäure gespalten.

\section{4-Diketo-tetrabydro-oxazol, $\mathrm{C}_{3} \mathrm{H}_{3} \mathrm{NO}_{3}$.}

Kocht man Isohydantoin mit alkoholisclier Salzsäure an Riickfluhkühler, so tritt zunächst klare Lösung ein; nach einiger Zeit erfolgt indessen Absch (idung ron Chlorammonium. Nach $1-1^{1} y$-stündigem Kochen liifot man abkühleu, treont die Flüssigkeit vom auskrystallisierten Salmiak unl lampit sie im Vakuum unter mehrfacher Erneuerung des Alkohols bis zum Vertreiben aller Salzsïure cin.

Lis verbleibt eine aus Diketo-tetrabydro-oxazol und Ammoniunehlorid bestehende farblose Krystallmasse, der man das erstere durch Betandeln mit Äther entziebt.

Das liewicht des nach dem Verdunsten des Äthers zurück1,eibenden Körpers beträgt $40 \%$ rom Gewichte des Ausgangsmaterials.

Läßt man die wäßrige Lösung des 2.4-Diketo-tetrabydro oxazols langsam verdunsten, so scheiden sicb wohlausgebildete lafelu ab, die bei $89-90^{\circ}$ zu einem farblosen Öl schmelzen, das seinerseits unter $11 \mathrm{~mm}$ Druck bei $173^{\circ}$ unzerserzt destilliert.

In Wasser, Alliobol und Essigester ist das 2.4-Diketo-tetrabydrooxazol sehr leicht Jöslich, etwas scbwerer in Äther; von Ligroin wird es fast gar nicht aufgenommen. Die wäßrige Lösung zeigt saure Reaktion.

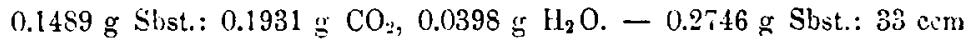
$\mathrm{N}\left(17^{\circ}, 75: \mathrm{mm}\right)$.

$$
\begin{aligned}
& \mathrm{C}_{3} \mathrm{IJ}_{3} \mathrm{O}_{3} \text { N. Ber. C 35.63, II 2.99, N 13.87. } \\
& \text { Gef. „ } 35.87, \text { 2.999, } 13.59 \text {. }
\end{aligned}
$$


Methyl-isubydantoin (2-Imino-4-keto-5-methyl-tetrahydrooxazol), $\mathrm{C}_{4} \mathrm{H}_{6} \mathrm{~N}_{2} \mathrm{O}_{2}$.

Vermischt man Milchsăureester mit einer konzentrierten, etwa j0prozentigen, alkoholischen Guanidialösung, so erfolgt unter lebbafter Erwärnung Abscheidung des Methyl-isohydantoius, die nach etwa 10-15 Stdu. heendet ist.

Die aus heibem Wasser umkrystallisierte Verbindung bildet irisierende Blättchen vom Schmp. $226^{\circ}$. Aus $12 \mathrm{~g}$ Milchsäureester erhält man etwa 8 g Methylisobydantoin, entsprechend $70 \%$ der theoretischen Ausbeute.

$0.1265 \mathrm{gg}$ Sbst.: $0.1870 \mathrm{~g} \mathrm{CO}_{2}, 0.0608 \mathrm{~g} \mathrm{H}_{2} \mathrm{O} .-0.0778 \mathrm{~g}$ Sbst.: $16.3 \mathrm{ccm}$ $\mathrm{N}\left(19^{\circ}, 761 \mathrm{~mm}\right)$.

$$
\begin{array}{ll}
\mathrm{C}_{4} \mathrm{H}_{6} \mathrm{~N}_{2} \mathrm{O}_{2} . & \text { Ber. } \mathrm{C} 42.10, \mathrm{H} 5.30, \mathrm{~N} 24.56 . \\
\text { Gef. } \$ 42.32, 》 5.64, 》 24.14 .
\end{array}
$$

Das Methyl-isohydantoin wird vou Wasser nur schwer benetzt, löst sich in der Hitze aber leicht darin. Von warmem Alkohol wird es ebenfalls leicht aufgenommen; scbwer löslich ist es in den meisten anderen organischen Lösungsmitteln.

Es löst sich ferner leicht in Säuren und wird aus einer solchen Lösung durch Neutralisieren mit Ammoniak oder Alkalien wieder abgeschieden. Aus heißer alkoholischer Salzsäure krystallisiert beim raschen Erkalten das Chlorbydrat des Methyl-isobydantoins in spitzen, zu Rosetten vereinigten Nädelchen aus.

Die Analyse dieses letzteren ergab:

$0.1781 \mathrm{~g}$ sbst.: $0.2069 \mathrm{~g} \mathrm{CO}_{2}, 0.075 \mathrm{~g} \mathrm{H}_{2} \mathrm{O} .-0.1090 \mathrm{~g}$ Sbst.: $17.4 \mathrm{ccm}$ $\mathrm{N}\left(17^{n}, 766\right) .-0.1260 \mathrm{~g}$ Sbst.: $0.1198 \mathrm{~g} \mathrm{Ag} \mathrm{Cl}$.

$\mathrm{C}_{4} \mathrm{H}_{6} \mathrm{~N}_{2} \mathrm{O}_{2}, \mathrm{HCl}$. B̈er. C 31.89, HI 4.68, N 18.62, Cl 2355.

Gef. » $31.68,>498, \otimes 18.67$, 23.51 .

2.4-Diketo-j-metbyl-tetrabydro-oxazol, $\mathrm{C}_{4} \mathrm{H}_{5} \mathrm{NO}_{3}$.

$10 \mathrm{~g}$ Mcthyl-isohydantoin wurden 1\%:2 Stunden mit einem Überschusse rerdünnter alkobolischer Salzsüure am Rückflußüüler gekocht. Es erfolgte z.uerst Lösung, balıl schieden sich indessen reichliche Mengen Cllorammonium $a b$, welche abfiltriert wurden. Das Filtrat wurde clarauf bis zum Verdampieo alles Chlorwasserstoffs eingeengt und der zum Teil noch aus salmiak bestehende Rückstand öfters mit $\ddot{A}$ ther extrahiert. Nach dem Trocknen über Natriumsulfat, Abfiltrieren und Abdestillieren des Lõsungsmittels verblieb eine klare sirupöse Flüssigkeit, deren Gewicht $32 \mathrm{~g}$ betrug und welche unter einem Druck von $15 \mathrm{~mm}$ bei $156-161^{\circ}$ überdestillicrte.

Durch Einstellen des Destillates in eine Kältemischung und andauerndes Reiben mit einem Glasstabe gelang es, den Sirup zum Krystallisieren zu bringen. Die Krystalle erwiesen sich indessen als stark hygroskopisch und z.crilossen beim Liegen an der Luft. Sie sinterten bei $39^{\circ}$ und waren hei $4+-45^{\circ}$ völlìg geschmolzen. 
Zur Analyse wurde dic Verbindung nochmals destilliert und der bei 169-170 unter einem Druck von 18-19 $\mathrm{mm}$ übergehende Anteil besonders aufgefangen. Trotzdem die Substanz sofort in Kügelchen gefüllt wurde, hatte sie bereits Wasser angezogen, so dab die Analysenresultate nicht genan stimmend ausfielen.

$0.1165 \mathrm{~g}$ Sbst.: $0.1732 \mathrm{~g} \mathrm{CO}, 0.0544 \mathrm{~g} \mathrm{H}_{2} \mathrm{O} .-0.1259 \mathrm{~g}$ Sbst.: $0.1920 \mathrm{~g}$ $\mathrm{CO}_{4}, 0.0559 \mathrm{~g} \mathrm{H}_{2} \mathrm{O} .-0.2675 \mathrm{~g}$ Sust. $: 28.2 \mathrm{~cm} \mathrm{~N}\left(21^{0}, 7.58 \mathrm{~mm}\right) .-0.1579 \mathrm{gr}$ Stst.: $16.2 \mathrm{ccm} \mathrm{N}\left(17^{\circ}, 755 \mathrm{~mm}\right)$.

$$
\begin{aligned}
& \mathrm{C}_{1} \mathrm{H}_{5} \mathrm{NO}_{3} \text {. Ber. C 41.72, } \mathrm{H} 4.3 \mathrm{~S}, \quad \mathrm{~N} \mathrm{12.17} \text {, } \\
& \text { Gef. » } 4055,41.59 \text {, » 5.2.2, 4.96, » 11.98, 11.83. }
\end{aligned}
$$

Das 2.4-Diketo-5-metbyl-tetrabydrooxazol ist sehr leicht löslich in Wasser, Alkohol, Äther und Essigester, sclwer löslich in Beñzol und Ligroin und scheidet sich aus allen diesen Solvenzien als Öl aus.

Phengl-isobydantoin (2-Imido-4-keto-5-phenyl-tetrabydrooxazol), $\mathrm{C}_{9} \mathrm{H}_{3} \mathrm{~N}_{2} \mathrm{O}_{2}$.

Turch Vermischen ron Mandels äureester mit einer äquivalenteo Menge Guavidin in konzentrierter alkoholiscber Lösung erhält man in einer fast 90-prozentigen Ausbeute das bei $256-257^{\circ}$ unter Zersetzung schmelzende Phenyl-isobydantoin.

Die Krystalle der Verbindung lösen sich ziemlich leicht in beißem Alkohol, schwerer in heißem Wasser und kommen beim Abküblen der Lösungsmittel fast vollständig wieder heraus.

$0.1015 \mathrm{~g}$ Sbst.: $0.2284 \mathrm{~g} \mathrm{CO}_{2}, 0.0417$ \& $\mathrm{H}_{2} \mathrm{O} .-0.1052 \mathrm{~g}$ Sbst.: $14.1 \mathrm{~cm}$. $\mathrm{N}^{*}\left(19^{\circ}, 7(i 1 \mathrm{~mm})\right.$.

$$
\begin{array}{ll}
\mathrm{C}_{9} \mathrm{H}_{8} \mathrm{~N}_{2} \mathrm{O}_{2} . & \text { Ber. C } 6134, \mathrm{H} 4.58, \mathrm{~N} 15.91 . \\
\text { Gej. " } 61.37, \text { » } 4.59 \text {, " } 15.44 .
\end{array}
$$

Das Nitrat des Phenylisohydantoins scheidet sich aus einer Anflïsun: des letzteren in verdünnter Salpetcrsăurc nach einigem Stehenlassen in Form prismatischer Kirystalle ab, welche sich bei $133^{\circ}$ zersetzen.

$0.1994 \mathrm{~g}$ Sbst.: $0.2464 \mathrm{~g} \quad \mathrm{OO}_{2}, 0.0 \% 36 \mathrm{gr} \mathrm{H}_{2} \mathrm{O} .-0.1519 \mathrm{~g}$ Shst.: $2.26 \mathrm{~cm}$ $\mathrm{N}\left(1 \mathrm{~S}^{0}, 762 \mathrm{~mm}\right)$.

$$
\begin{aligned}
& \mathrm{C}_{9} \mathrm{H}_{8} \mathrm{~N}_{2} \mathrm{O}_{2}, \mathrm{HNO}_{3} \text {. Ber. C 45.17, If 3.79, } \mathrm{N} 17.5 \mathrm{~S} \text {. } \\
& \text { Gef. » 44.98, »4.01, 》17.2.2. }
\end{aligned}
$$

j-Pbenyl-2.4-diketo-tetrahydro-oxazol, $\mathrm{C}_{9} \mathrm{H}_{i} \mathrm{NO}_{3}$.

Zur Überführung in das j-Phenyl-2.4-diketo-tetrahydro-oxazol werden $3 \mathrm{~g}$ Phenyl-isolhydantoin mit etwa $50 \mathrm{ccm}$ verdünnter Salzsäure übergossea und auf dem Wasserbade erwämt. Zunächst geht alles in Lösung, bakd aber setzen sich am Boden des GefißBes schwach gelblich gelärbte öltröpfchen ab und beim darauf folgenden Erkalten erfült sich die ganze Flüssigkeit mit farlolusen, grobblattrigen-Kírystallen des Phengl-diketo-tetrahydro-oxazols.

biesc werden abgesaugt, mit Wasser gewaschen und dann aus etwa de? 15-fachen Meuge heißen Wassers umirrstallisiert. 
Die Verbindung wird so in glänzenden Blättchen erhalten, deren Schmelzpunkt bei $108^{\circ}$ liegt und welche sich in Alkobol, Äther, Essigester leicht, schwerer in Ligroin und Benzol lösen.

$0.1318 \mathrm{~g}$ Sbst.: $0.2950 \mathrm{~g} \mathrm{CO}_{2}, 0.0517 \mathrm{~g} \mathrm{H}_{2} \mathrm{O} .-0.0921 \mathrm{~g}$ Sbst.: $6.6 \mathrm{ccm}$ $\mathrm{N}\left(20^{\circ}, 763 \mathrm{~mm}\right)$.

$$
\begin{aligned}
& \mathrm{C}_{9} \mathrm{H}_{7} \mathrm{NO}_{3} . \text { Ber. C } 61.00, \mathrm{H} 3.98, \mathrm{~N} 7.91 . \\
& \text { Gef. } \$ 61.04, ~ 4.38, \$ 8.05 .
\end{aligned}
$$

Durch Kochen mit Baryt wird das 5-Phenyl-2.4-diketo-tetrabydrooxazol in Mandelsäure, Ammoniak und Koblendioxyd gespalten.

[Oxy-methyl]-isobydantoin (2-Imino-4-keto-5-[oxy-methyl]tetrahydro-oxazol), $\mathrm{NH}: \mathrm{C}<\mathrm{NH} . \mathrm{CO}$

$3 \mathrm{~g} \mathrm{Glycerinsäure-methylester} \mathrm{wurden} \mathrm{in} \mathrm{absolut} \mathrm{methylalkoho-}$ liseher Lösung mit der ăçuivalenten Menge Cruanidin -- gleichfalls in Methylalkohol gelöst - versetzt, wobei nach kurzer Zeit infolge der Reaktiouswärme der Alkobol ins Sieden geriet. Aus der resultierenden dicken öligen Masse schieden sich im Verlauf mehrerer Tage Krystalle ab, die scharf abgesaugt, mit wenig absolutem Alkohol gewaschen und dann auf T'on gebrasht wurden.

Durch Umkrystallisieren aus sehr wenig Wasser erhielt man kleine Prismen vom Schmp. $197^{\circ}$.

Aus der angewandten Menge des Glycerinsäureesters wurden so nur $0.5 \mathrm{~g}$ reines Oxymethyl-isobydantoin erhalten; etwas gesteigert wurde die Ausbeute, als man in äthylalkoholischer Lösung arbeitete.

Die Verbindung ist sehr leicht löslich in Wasser, etwas schwerer is Albobol und sehr schwer in den anderen gewöbnlichen organischen Iösungsmitteln.

$0.1264 \mathrm{~g}$ Sbst.: $0.1708 \mathrm{~g} \mathrm{CO}_{2}, 0.05 .54 \mathrm{~g} \mathrm{H}_{2} \mathrm{O} .-0.0961 \mathrm{~g}$ Sbst.: $18.0 \mathrm{ccm}$ N $\left(23^{n}, 758 \mathrm{~mm}\right)$.

$$
\begin{array}{ll}
\mathrm{C}_{4} \mathrm{H}_{6} \mathrm{~N}_{2} \mathrm{O}_{3} . & \text { Ber. } \mathrm{C} 36.92, \mathrm{HI} 4.61, \mathrm{~N} 21.54 . \\
& \text { Gef. } \$ 36.55, \$ 4.90, \$ 21.10 .
\end{array}
$$

Glykocyamidin aus Glykokollester und Guanidiu.

Vermischte man Glykokollester mit der äquiralenten Nenge freien Guanirlins, so trat lebhafte Reaktion ein, in deren Verlanf reichliche Abspaltung von Ammoniak zu bemerken war, und schließlich erstarrte das Gemisch zu einem dicken Krystallbrei.

Der nach dem Abpressen auf Ton aus wenig Wasser umkrystallisierte Körper erwies sich als Glykocyamidin sowohl durch sein Verhalten gegenũber ammoniakalischer Silberlösung, Quecksilberchlorid-, Zinkcblorid-, Kupferacetat- und Ferrichlorid-Lüsung, als auch durch die Analyse. 
$0.1712 \mathrm{~g}$ Sbst.: $0.2292 \mathrm{~g} \mathrm{CO}, 0.0813 \mathrm{~g} \mathrm{H}_{2} \mathrm{O} .-0.1590 \mathrm{~g}$ Sbst.: $57.6 \mathrm{ccm}$ I $\left(15^{0}, 748 \mathrm{~mm}\right)$.

$$
\begin{aligned}
& \mathrm{C}_{-3} \mathrm{H}_{5} \mathrm{~N}_{3} \text { O. Ber. C 36.36, H 5.05, N 42.42. } \\
& \text { Gef. 》 } 36.51 \text {, 》5.31, " } 41.70 \text {. }
\end{aligned}
$$

[äthoxy-acetyl]-guanidin und [Äthoxy-propionyl]-guanidin.

It quivalente Mengen des nach der Methode von Schreiner dargestellten it hoxy-essigesters und Guanidins wurden in konzentrierter absolut alkoholischer Lösung gemischt, worauf alsbald starke Erwärmung, aber keine Abspaltung von Ammoniak wahrzuuebmen war. Nach wenigen Stunden war

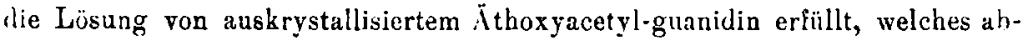
filtriert und für die Analyse ans Alkohol umkrystallisiert wurde.

$0.1144 \mathrm{~g}$ Sbst.: $0.1744 \mathrm{~g} \mathrm{CO}_{2}, 0.0789 \mathrm{~g} \mathrm{H}_{3} \mathrm{O}$. $-0.1372 \mathrm{~g}$ Sbst.: 35.2 cem $\mathrm{N}\left(26^{0}, 763 \mathrm{~mm}\right)$.

$$
\begin{array}{ll}
\mathrm{C}_{5} \mathrm{H}_{1} \mathrm{~N}_{3} \mathrm{O}_{2} . & \text { Ber. C 41.3s, H 7.60, N } 29.03 . \\
& \text { Gel. 》41.56, " 7.71, 》28.91. }
\end{array}
$$

Das Äthoxyacetyl-guanidin ist leicht löslich in Wasser und heißem Alkobol, schwer löslich in Aceton und fast unlöslich in Äther und Benzol. Die wäßrige Lösung zeigt alkalische Reaktion. Der Schmelzpunkt des $\tilde{A}$ thoxyacetyl-guanidins liegt bei $162^{\circ}$.

Tas Ätboxypropiongl-guanidin wurde durch Einwirkung ron Guanidin auf Äthoxy-propionsäureester in konzentrierter alkoholischer Jösung erbalten und entstand dabei in einer Ausbeute, die $80 \%$ der theoretisch berechneten entspricht. Die Verbindung schunilat bei $196^{\circ}$ und entspricht in ihrem Verbalten gegenüber Lösungsmitteln ungefähr dem Ätboxyacetyl-guanidin.

$0.1505 \mathrm{~g}$ Sbst.: $0.24 \$ 8 \mathrm{~g} \mathrm{CO}_{2}, 0.1120 \mathrm{~g} \mathrm{H}_{2} \mathrm{O} .-0.1351 \mathrm{~g}$ Sbst.: $30.2 \mathrm{ccm}$ $\mathrm{N}(2000,76.7 \mathrm{~mm})$.

$$
\begin{array}{ll}
\mathrm{C}_{6} \mathrm{H}_{13} \mathrm{~N}_{3} \mathrm{O}_{2} . & \text { Ber. C } 45.28, \mathrm{H} \\
& \text { Gef. } 4.17, \mathrm{~N} 26.41 .
\end{array}
$$

\section{C. Liebermann: Erwiderung an Hrn. A. Kronstein.}

(Eingegangen am 17. Juni 1913.)

In eiver in der vorletzten Nummer dieser Berichte (S. 1812) erschienenen Notiz: »Zur Kenutnis der Polymerisation« beschwert sich Hr. A. Kronstein darüber, da $B$ M. Kardos und ich in unserer letzten Abhandlung über Polyzimtsäure ${ }^{1}$ ) in sein Arbeitsgebiet einyegriffen und seine früberen Arbeiten über diesen Gegenstand nicht genügeud berïcksichtigt bätten.

1) B. 46, 1055 [1913]. 